\title{
Modified Rio Score with Platform Therapy Predicts Treatment Success with Fingolimod and Natalizumab in Relapsing-Remitting Multiple Sclerosis Patients
}

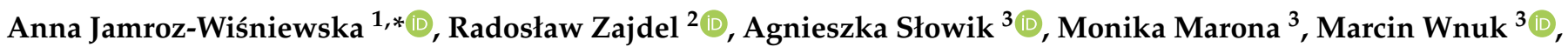 \\ Monika Adamczyk-Sowa ${ }^{4}$, Bożena Adamczyk ${ }^{4}$, Anetta Lasek-Bal ${ }^{5}$, Przemysław Puz ${ }^{5}$, Arkadiusz Stęposz ${ }^{5}$, \\ Ewa Krzystanek ${ }^{6}$, Maja Patalong-Ogiewa ${ }^{7}$, Anna Pokryszko-Dragan ${ }^{8}$, Sławomir Budrewicz ${ }^{8}$, \\ Dorota Koziarska ${ }^{9}{ }^{(1)}$, Anna Karbicka ${ }^{10}$, Sławomir Wawrzyniak ${ }^{11}$, Waldemar Fryze ${ }^{12}$, \\ Marzena Furtak-Niczyporuk ${ }^{13}$ and Konrad Rejdak ${ }^{1}$ (D)
}

check for updates

Citation: Jamroz-Wiśniewska, A.; Zajdel, R.; Słowik, A.; Marona, M.; Wnuk, M.; Adamczyk-Sowa, M.; Adamczyk, B.; Lasek-Bal, A.; Puz, P.; Stęposz, A.; et al. Modified Rio Score with Platform Therapy Predicts Treatment Success with Fingolimod and Natalizumab in Relapsing-Remitting Multiple Sclerosis Patients. J. Clin. Med. 2021, 10, 1830. https://doi.org/ $10.3390 /$ jcm10091830

Academic Editor: Cristoforo Comi

Received: 3 March 2021

Accepted: 20 April 2021

Published: 22 April 2021

Publisher's Note: MDPI stays neutral with regard to jurisdictional claims in published maps and institutional affiliations.

Copyright: (c) 2021 by the authors. Licensee MDPI, Basel, Switzerland. This article is an open access article distributed under the terms and conditions of the Creative Commons Attribution (CC BY) license (https:/ / creativecommons.org/licenses/by/ $4.0 /)$.
1 Department of Neurology, Medical University of Lublin, Jaczewskiego 8, 20-054 Lublin, Poland; krejdak@europe.com

2 Chair of Informatics in Business, University of Lodz, Rewolucji 1905 Roku 37/39, 91-001 Lodz, Poland; radoslaw.zajdel@interforum.pl

3 Department of Neurology, Collegium Medicum, Jagiellonian University, Jakubowskiego 2, 30-688 Krakow, Poland; agnieszka.slowik@uj.edu.pl (A.S.); monika.marona@interia.pl (M.M.); marcin.a.wnuk@gmail.com (M.W.)

4 Department of Neurology, School of Health Sciences in Zabrze, Medical University of Silesia in Katowice, 3-go Maja 13-15, 41-800 Zabrze, Poland; msowa@sum.edu.pl (M.A.-S.); badamczyk@sum.edu.pl (B.A.)

5 Department of Neurology, School of Health Sciences, Medical University of Silesia in Katowice, Ziolowa 45-47, 40-635 Katowice, Poland; alasek@gcm.pl (A.L.-B.); ppuz@gcm.pl (P.P.); asteposz@gcm.pl (A.S.)

6 Department of Neurology, Faculty of Medical Sciences in Katowice, Medical University of Silesia, Medykow 14, 40-752 Katowice, Poland; ewa26@mp.pl

7 Department of Neurorehabilitation, Faculty of Medical Sciences in Katowice, Medical University of Silesia, Medykow 14, 40-752 Katowice, Poland; mpatalongogiewa@sum.edu.pl

8 Department of Neurology, Wroclaw Medical University, Borowska 213, 50-566 Wroclaw, Poland; annapd@interia.pl (A.P.-D.); slawomir.budrewicz@umed.wroc.pl (S.B.)

9 Department of Neurology, Pomeranian Medical University in Szczecin, Unii Lubelskiej 1, 71-252 Szczecin, Poland; synaps3@wp.pl

10 Department of Neurology, Regional Hospital, Arkonska 4, 71-455 Szczecin, Poland; anuszek-4@o2.pl

11 Department of Neurology, 10th Military Research Hospital and Polyclinic, Powstancow Warszawy 5, 85-681 Bydgoszcz, Poland; swawrzyniak@wp.pl

12 Department of Neurology, Copernicus Pl, M. Kopernik Hospital, Nowe Ogrody 1-6, 80-803 Gdansk, Poland; w.fryze@wp.pl

13 Department of Public Health, Medical University of Lublin, 20-954 Lublin, Poland; marzenafurtakniczyporuk@umlub.pl

* Correspondence: anna.jamroz@umlub.pl; Tel.: +48-81-72-44-720

Abstract: Background: Reliable markers of disease outcomes in multiple sclerosis (MS) would help to predict the response to treatment in patients treated with high efficacy drugs. No evidence of disease activity (NEDA) has become a treatment goal whereas the modified Rio score (MRS) predicts future suboptimal responders to treatment. The aim of our study was to identify factors that would predict poor response to treatment with natalizumab and fingolimod. Methods: In the multicenter prospective trial, 336 subjects were enrolled, initiating therapy with natalizumab $(n=135)$ or fingolimod ( $n=201)$. Data on relapse rate, the expanded disability status scale, and MRI results were collected, and MRS was estimated. Results: NEDA-3 after the first year of therapy was $73.9 \%$ for natalizumab and $54.8 \%$ for fingolimod $(p<0.0001)$. Patients with MRS $=0$ in the last year on platform therapy had the best NEDA-3 (71\%) and patients with MRS $=3$ had the worst NEDA-3 (41\%) in the first year of treatment with the second-line therapy. Conclusion: We conclude that switching to the second-line therapy should occur earlier to enable better results for patients treated with natalizumab or fingolimod. The outcome on both drugs is better with better neurological conditions and lower MRS of the patient on the platform therapy. 
Keywords: relapsing-remitting type of multiple sclerosis; no evidence of disease activity (NEDA); modified Rio score (MRS); natalizumab; fingolimod

\section{Introduction}

Multiple sclerosis (MS) is an autoimmune neurological disease that is a chronic, progressive disability and perceived as incurable. Nevertheless, there are modern and more aggressive drugs used in the treatment of relapsing-remitting MS (RR-MS) that prevent the development of disability and treat the disease with higher efficacy than others. Among many currently available compounds, fingolimod and natalizumab belong to high efficacy drugs with the longest experience in clinical practice. They are used in case of inefficacy of the first-line drugs such as interferon-beta, glatiramer acetate, teriflunomide, or dimethyl fumarate. Fingolimod (a sphingosine-receptor agonist) and natalizumab (a humanized monoclonal antibody against the cell adhesion molecule $\alpha 4$-integrin) are used when MS becomes highly active because the possible side effects associated with their use are more serious (including progressive multifocal leukoencephalopathy associated mainly with natalizumab use) [1]. This is the so-called escalation therapy approach in contrast to induction therapy, used from the beginning of disease onset (i.e., treatment with immunosuppressive drugs like alemtuzumab, cladribine, mitoxantrone, or autologous-hematopoietic stem cell transplantation, followed by the long-term use of maintenance therapy with diseasemodifying treatment, DMT) used especially in case of rapidly evolving or severe MS (RES) [2].

No evidence of disease activity (NEDA) is a main goal of the MS treatment strategy. NEDA-3 includes the absence of relapses, no evidence of magnetic resonance (MR) activity (no new or enlarged T2 lesions or new contrast-enhancing T1 lesions), and no progression of disability (no confirmed expanded disability status scale, EDSS, worsening) in the previous 6 months. NEDA-3 reflects mainly focal inflammatory activity measures, while NEDA-4 incorporates a neurodegenerative processes indicator, i.e., progression of disability unrelated to clinical relapse. In the case of NEDA- 4 , lack of brain volume loss $>0.4 \%$ is also added [3,4]. Another indicator of NEDA, NEDA-5 additionally includes the level of cerebrospinal fluid neurofilament light chain as a biomarker of neurodegeneration in MS [5]. There is also a term, MEDA, indicating minimal evidence of disease activity such as marginal MRI activity (one to two new T2 lesions), in the absence of relapses and contrast-enhancing lesions [6].

With the new drugs, the achievement of NEDA status lasting a few years seems to be more and more likely. The NEDA-3 for natalizumab in the AFFIRM trial was 37\% [7], whereas for fingolimod in the FREEDOMS trial was 33\% and NEDA-4 in the pooled FREEDOMS/FREEDOMS II population was 19.7\% [8,9].

In order to achieve NEDA, it would be optimal to identify factors early on that influence the response to treatment. A clinical score that predicts the response in interferon beta-treated patients was proposed by Rio et al. and then modified by Sormani et al. [10,11]. The Rio score combines the assessment of 1 year of clinical relapses, disability progression (measured by the EDSS), and active MRI lesions [10]. The modified Rio score (MRS) includes the combination of the MRI activity and clinical relapses, as shown in Table 1 [11]. Both scores are used to identify patients with poor outcomes during subsequent years. The Rio score was also assessed in MS patients treated with natalizumab [12]. 
Table 1. Modified Rio score scoring criteria [11].

\begin{tabular}{|c|c|c|}
\hline Criterion & Change over 1st Year & Score \\
\hline \multirow{2}{*}{ MRI } & $\leq 4$ new $\mathrm{T} 2$ lesions & 0 \\
\hline & $>4$ new T2 lesions & 1 \\
\hline \multirow{3}{*}{ Relapse } & No relapses & 0 \\
\hline & 1 relapse & 1 \\
\hline & $\geq 2$ relapses & 2 \\
\hline
\end{tabular}

The study aimed to identify factors assessed during initial platform therapy correlating with treatment success after switching to high efficacy drugs like natalizumab and fingolimod in the first year of treatment.

\section{Materials and Methods}

\subsection{Subjects and Study Design}

Data were collected from 336 patients initiating second-line therapy, natalizumab or fingolimod, between 2013 and 2017 in ten Polish MS centers. This was a multicenter prospective trial. Patients qualified to the treatment with natalizumab or fingolimod had to fulfil the following criteria: age above 18 years old, diagnosis of RR-MS according to the 2010 revisions of the McDonald criteria of MS [13], treatment with first-line drugs during at least one year, at least two moderate relapses requiring treatment with steroids (increase in EDSS score of 1-2 points or increase of 2 points in one or two functional systems or increase of 1 point in at least 4 functional systems) or at least one severe relapse (with increase in EDSS higher than in definition of a moderate relapse) in the previous year of treatment, and at least 2 gadolinium-enhancing lesions or at least three new T2-lesions on control MRI. According to national health fund regulations, patients with the presence of antibodies against the John Cunningham virus (JCV) in their serum could not be qualified to the treatment with natalizumab. Apart from this, the choice of the second-line drug was left to the individual center. All the eligible patients were recruited.

Exclusion criteria included a clinical course different than RR-MS and RES in naïve patients whose first DMT was fingolimod or natalizumab.

Data on relapse rate and EDSS were prospectively documented at routine clinic visits performed every three months. EDSS data were collected until December 2018. An MRI was routinely performed before treatment and every year. A standardized brain MRI protocol with gadolinium was used including T2-weighted, fluid-attenuated inversion recovery (FLAIR), diffusion-weighted imaging (DWI) and pre- and postcontrast T1-weighted sequences, at magnetic field strengths of 1.5 T. Data were retrospectively collected for the purpose of this study. On the basis of MRI and relapses, MRS was estimated.

We assessed the effectiveness of treatment in two ways. First, we calculated NEDA-3 for the first year of therapy in the group of patients that started treatment with fingolimod or natalizumab. Second, we estimated NEDA-3 in the first year of therapy with the secondline drugs depending on MRS in the last year on platform therapy before switching to the second-line therapy.

\subsection{Statistical Analysis}

The results were analyzed with the use of statistical methods, including some multidimensional tests. The Shapiro-Wilk test was used to assess normal distribution. The studied characteristics with non-normal distribution as well as qualitative and quantitative data were analyzed with the use of non-parametric tests, including the Kruskal-Wallis, ANOVA, Pearson's Chi-squared, and Mann-Whitney U tests. General descriptive statistics methods were also used. The statistically significant $p$ level was set at $<0.05$. 


\section{Results}

\subsection{Participant Characteristics}

There were 336 patients in the whole group, including 222 females $(66.1 \%)$ and 114 males (33.9\%). Of the 336 patients, 201 were treated with fingolimod and 135 with natalizumab. Patients were treated previously with the first-line drugs, most of them with interferons beta ( $82 \%), 16 \%$ with glatiramer acetate, and only $2 \%$ with other drugs (dimethyl fumarate and teriflunomide, because they were not refunded in Poland at the time of study). There were no dropouts in the first year of treatment (that was taken into consideration). All patients completed at least one year of therapy with fingolimod or natalizumab in order to be included into the analysis. Characteristics of patients are given in Table 2. We found no statistically significant differences between the two cohorts of patients at baseline.

Table 2. Baseline demographic and clinical characteristic of patients treated with fingolimod and natalizumab.

\begin{tabular}{|c|c|c|c|c|}
\hline Characteristic & All Patients $n=336$ & Fingolimod & Natalizumab & $p$ (Mann-Whitney U) \\
\hline Age, years, mean (SD) & $38.7(10.0)$ & $39.4(10.5)$ & $37.6(9.0)$ & 0.133 \\
\hline Female, $n(\%)$ & $222(66.1)$ & $135(67.16)$ & $87(64.4)$ & $p=0.15$ \\
\hline Onset age, years (SD) & $27.5(9.2)$ & $27.6(9.8)$ & $27.2(8.1)$ & 0.995 \\
\hline $\begin{array}{c}\text { Disease duration until } \\
\text { 1/November/2018, years (SD) }\end{array}$ & $10.7(5.4)$ & $11.2(5.7)$ & $9.8(4.7)$ & 0.094 \\
\hline $\begin{array}{l}\text { Mean ARR before initiation of } \\
\text { treatment, number (SD) }\end{array}$ & $2.0(0.6)$ & $2.0(0.7)$ & $2.1(0.6)$ & 0.206 \\
\hline $\begin{array}{l}\text { Mean EDSS before initiation of } \\
\text { treatment, points (SD) }\end{array}$ & $3.26(1.3)$ & $3.3(1.3)$ & $3.2(1.4)$ & 0.598 \\
\hline $\begin{array}{c}\text { Number of T2 lesions on MRI } \\
\text { one year before initiation of } \\
\text { treatment, number (SD) }\end{array}$ & $19.4(6.2)$ & $19.3(6.5)$ & $19.6(5.6)$ & 0.455 \\
\hline $\begin{array}{l}\text { Number of Gd+ lesions on } \\
\text { MRI one year before initiation } \\
\text { of treatment, number (SD) }\end{array}$ & $2.3(3.7)$ & $2.2(3.4)$ & $2.5(4.1)$ & 0.366 \\
\hline Patients treated & 336 & $201(59.8)$ & 135 (40.2) & \\
\hline
\end{tabular}

ARR, annualized relapse rate; EDSS, expanded disability status scale; Gd+, gadolinium; MRI, magnetic resonance imaging; SD, standard deviation.

\subsection{No Evidence of Disease Activity 3 (NEDA-3) Results in Patients Treated with Natalizumab and Fingolimod}

3.2.1. NEDA-3 in the Group of Patients that Started Treatment with Fingolimod or Natalizumab

After the first year of therapy $71.3 \%$ of patients treated with natalizumab and $53.9 \%$ of patients treated with fingolimod attained NEDA-3 (Table 3).

3.2.2. NEDA-3 in the First Year of Therapy with the Second-Line Drugs Depending on MRS in the Last Year on Platform Therapy

We assessed NEDA-3 in the first year of treatment with fingolimod and natalizumab depending on MRS one year before switching to the second-line treatment. Patients on platform therapy with MRS $=0$ one year before switching to the second-line treatment in $71 \%$ attained NEDA-3 in the first year of treatment with natalizumab or fingolimod. Of the patients with MRS $=1,62 \%$ had NEDA-3; of the patients with MRS $=2,65 \%$ attained NEDA-3; whereas of the patients with MRS $=3$, only $41 \%\left(\mathrm{Chi}^{2}\right.$ Pearson $p=0.05248$, $\mathrm{R}$ Spearman $p=0.02743$ ) had NEDA-3 in the first year of treatment with natalizumab or fingolimod-Table 4 . There were no significant differences between patients using interferon beta or glatiramer acetate. 
Table 3. NEDA-3 for fingolimod and natalizumab in the first year of treatment for patients that began the treatment in 2013 and 2014.

\begin{tabular}{|c|c|c|c|}
\hline$n=281$ & & NEDA-3 in the First Year of Treatment $n(\%)$ & $p$ \\
\hline \multirow{2}{*}{ All } & NEDA-3 & 169 & \\
\hline & No-NEDA & 112 & \\
\hline \multirow{2}{*}{ Fingolimod } & NEDA-3 & $97(53.9)$ & \multirow{4}{*}{$p<0.0001$} \\
\hline & No-NEDA & $83(46.1)$ & \\
\hline \multirow{2}{*}{ Natalizumab } & NEDA-3 & $72(71.3)$ & \\
\hline & No-NEDA & $29(28.7)$ & \\
\hline
\end{tabular}

NEDA, no evidence of disease activity; available data from $n=281$.

Table 4. Relationship between MRS on platform therapy before switching to the second-line treatment and NEDA-3 in the first year of treatment with natalizumab or fingolimod.

\begin{tabular}{ccccccc}
\hline & MRS = 0 & MRS = 1 & MRS = 2 & MRS = 3 & $\begin{array}{c}\text { Chi }^{2} \text { Pearson } \\
\text { R Spearman }\end{array}$ \\
\hline \multirow{2}{*}{ All } & NEDA-3 & $5(71.4 \%)$ & $10(62.5 \%)$ & $118(65.9 \%)$ & $14(41.2 \%)$ & $p=0.05248$ \\
& No-NEDA-3 & $2(28.6 \%)$ & $6(37.5 \%)$ & $61(34.1 \%)$ & $20(58.8 \%)$ & $p=0.02743$ \\
\hline
\end{tabular}

IFNs, interferons; MRS, modified Rio score; NEDA, no evidence of disease activity.

\section{Discussion}

Reliable markers of disease outcomes in MS would help to predict the response to treatment in patients treated with second-line drugs who may continue to have disease activity. NEDA and MEDA have become a treatment goal and outcome measure [3]. MRS is another marker that helps to detect future suboptimal responders to treatment [11].

In our study, the higher proportion of NEDA-3 in the first year was associated with natalizumab compared to fingolimod; nevertheless, for both drugs it was very good$73.9 \%$ and $54.8 \%$ respectively. In a few studies, these two drugs were compared. In the second-Line GATE study, it was found that after 12 months of treatment, ARR was more reduced by natalizumab compared to fingolimod [14]. In another study, a higher proportion of patients treated with natalizumab (55.8\%) compared to fingolimod (11.6\%) achieved NEDA-3, and cortical lesions status showed the higher efficacy of natalizumab versus fingolimod in suppressing disease activity in RR-MS patients [3]. In an Italian study, after two years of treatment, natalizumab proved higher efficacy in NEDA-3 (39\%) in comparison to fingolimod (22\%), and confirmed regression of disability was higher in the case of natalizumab (19.2\% vs. 6.7\%) [15]. Baroncini et al. showed higher NEDA-3 for natalizumab (70\%) than fingolimod (44\%) after two years of follow-up without differences in disability worsening [16]. Similarly, in the Preziosa study, the natalizumab group had a higher proportion of freedom from MRI activity ( $67 \%$ vs. $36 \%$ in fingolimod group) and NEDA-3 (57\% vs. $28 \%$ ) [17]. In a study carried out in the Polish population, more patients under natalizumab had NEDA-3 after two and four years of follow-up compared to fingolimod $(66.2 \%$ and $42.1 \%$ vs. $52.1 \%$ and $29.5 \%$, respectively) [18].

We found an association between MRS in the year preceding the switch to the secondline drugs and NEDA-3 in the first year of therapy with these drugs (i.e., natalizumab and fingolimod). Better results were achieved with $\mathrm{MRS}=0, \mathrm{MRS}=1, \mathrm{MRS}=2$-more patients had NEDA-3 later $(71.4 \%, 62.5 \%$, and $65.9 \%$, respectively) comparing to MRS $=3$ (only $41.2 \%)$. MRS in the year before initiation of treatment with the second-line drugs had a significant effect on disability progression, relapses, and new T2 lesions on MRI in the first year of natalizumab or fingolimod treatment. This suggests better effects of the second-line drugs in patients with a lower activity of disease in the last year on platform therapy.

This means that a better outcome could be expected in patients that have fewer relapses and fewer new demyelinating lesions on the MRI before changing to a more aggressive treatment. Neurologists should not wait too long; it is better to aggravate 
therapy when a patient has moderate disease activity than wait until a severe course of the disease that would lead to more relapses, disability progression, and MRI activity on more potent drugs in the future.

Raffel and co-authors found that relapses and MRS in the first year of natalizumab treatment predicted only year 1 and year 2 EDSS progression, and that effect disappeared with a longer observation period [12]. It suggested a disconnect between natalizumab's ability to suppress the focal inflammatory activity underlying relapses and new T2 MRI lesions, and its putative effect on the progression of long-term disability [12]. In an adjusted analysis by Kapica-Topczewska et al., a higher baseline EDSS was a predictor of relapse $(p<0.001)$ and NEDA-3 failure [18].

There were no statistically significant differences between natalizumab and fingolimod regarding the probability of no relapse, depending on a MRS higher than 0 .

A limitation of this analysis is the loss of patients in the follow-up period. This study had some methodological shortcomings due to its real-world evidence (RWE) nature. Follow-up MRI scans of MS patients were not always performed in daily clinical practice; therefore, the data after 5 years of study are limited. Nevertheless, this is the first report of predictive treatment response in patients with RR-MS treated with natalizumab and fingolimod after the switch from platform therapy.

\section{Conclusions}

As patients with MRS $=0$ in the last year on platform therapy had the best NEDA $(71 \%)$ and patients with MRS $=3$ had the worst NEDA $(41 \%)$ in the first year of treatment with the second-line therapy, we conclude that switching to the second-line therapy should occur earlier to enable better results for the patients treated with natalizumab or fingolimod. We should not wait too long as patients with higher MRS on interferon beta or glatiramer acetate would have a worse outcome on second-line drugs. If a patient has both radiological activity ( 4 or more new T2 lesions) and clinical activity ( 2 or more relapses), then it is far less probable that such a patient has the status of NEDA in the first year of treatment with natalizumab or fingolimod. On the other hand, if we switch a patient with some radiological activity but with only one relapse or with even better neurological status (e.g., without new demyelinating lesions or without relapses), the outcome on a new drug would be better.

Comparing the results of both drugs, we can say they both suppress disease activity (natalizumab a bit better), but the outcome on both drugs is better with a better neurological condition of the patient on platform therapy.

In conclusion, the evaluation of MRS in daily clinical practice in patients on platform therapy is useful to predict the response to natalizumab/fingolimod treatment and would be helpful to optimize MS therapy. It is recommended to switch patients with a lower MRS to achieve a better outcome.

Author Contributions: Conceptualization, A.J.-W. and K.R.; methodology, A.J.-W., K.R., and R.Z.; software, R.Z.; validation, A.J.-W., K.R., and R.Z.; formal analysis, A.J.-W., K.R., and R.Z.; investigation, A.J.-W., R.Z., A.S. (Agnieszka Słowik), M.M., M.W., M.A.-S., B.A., A.L.-B., P.P., A.S. (Arkadiusz Stẹposz), E.K., M.P.-O., A.P.-D., S.B., D.K., A.K., S.W., W.F., and K.R.; resources, A.J.-W., R.Z., A.S. (Agnieszka Słowik), M.M., M.W., M.A.-S., B.A., A.L.-B., P.P., A.S. (Arkadiusz Stepposz), E.K., M.P.-O., A.P.-D., S.B., D.K., A.K., S.W., W.F., M.F.-N., and K.R.; data curation, A.J.-W., R.Z., A.S. (Agnieszka Słowik), M.M., M.W., M.A.-S., B.A., A.L.-B., P.P., A.S. (Arkadiusz Stęposz), E.K., M.P.-O., A.P.-D., S.B., D.K., A.K., S.W., W.F., and K.R.; writing—original draft preparation, A.J.-W., K.R., and R.Z.; writing-review and editing, A.J.-W., R.Z., A.S. (Agnieszka Słowik), M.M., M.W., M.A.-S., B.A., A.L.-B., P.P., A.S. (Arkadiusz Stęposz), E.K., M.P.-O., A.P.-D., S.B., D.K., A.K., S.W., W.F., M.F.-N., and K.R.; visualization, A.J.-W., R.Z., A.S. (Agnieszka Słowik), M.M., M.W., M.A.-S., B.A., A.L.-B., P.P., A.S. (Arkadiusz Stęposz), E.K., M.P.-O., A.P.-D., S.B., D.K., A.K., S.W., W.F., M.F.-N., and K.R.; supervision, K.R.; project administration, A.J.-W., K.R., and R.Z. All authors have read and agreed to the published version of the manuscript.

Funding: This research received no external funding. 
Institutional Review Board Statement: The study was conducted in accordance with the Declaration of Helsinki, and the protocol was approved by the Ethics Committee of Medical University of Lublin (project identification code 22/2011).

Informed Consent Statement: All subjects gave their informed consent for inclusion before they participated in the study.

Data Availability Statement: The data presented in this study are available on request from the corresponding author. The data are not publicly available due to ethical restrictions.

Acknowledgments: We thank all our patients for their participation in the study.

Conflicts of Interest: The authors declare no conflict of interest.

\section{References}

1. Oshima, Y.; Tanimoto, T.; Yuji, K.; Tojo, A. Drug-associated progressive multifocal leukoencephalopathy in multiple sclerosis patients. Mult. Scler. J. 2018, 25, 1141-1149. [CrossRef] [PubMed]

2. Pardo, G.; Jones, D.E. The sequence of disease-modifying therapies in relapsing multiple sclerosis: Safety and immunologic considerations. J. Neurol. 2017, 264, 2351-2374. [CrossRef] [PubMed]

3. Puthenparampil, M.; Cazzola, C.; Zywicki, S.; Federle, L.; Stropparo, E.; Anglani, M.; Rinaldi, F.; Perini, P.; Gallo, P. NEDA-3 status including cortical lesions in the comparative evaluation of natalizumab versus fingolimod efficacy in multiple sclerosis. Ther. Adv. Neurol. Disord. 2018, 11. [CrossRef] [PubMed]

4. $\quad$ Beadnall, H.N.; Wang, C.; Van Hecke, W.; Ribbens, A.; Billiet, T.; Barnett, M.H. Comparing longitudinal brain atrophy measurement techniques in a real-world multiple sclerosis clinical practice cohort: Towards clinical integration? Ther. Adv. Neurol. Disord. 2019, 12, 1-20. [CrossRef] [PubMed]

5. Giovannoni, G.; Nath, A. After the storm: Neurofilament levels as a surrogate endpoint for neuroaxonal damage. Neurology 2011, 76, 1200-1201. [CrossRef] [PubMed]

6. Prosperini, L.; Mancinelli, C.; Haggiag, S.; Cordioli, C.; De Giglio, L.; De Rossi, N.; Galgani, S.; Rasia, S.; Ruggieri, S.; Tortorella, C.; et al. Minimal evidence of disease activity (MEDA) in relapsing-remitting multiple sclerosis. J. Neurol. Neurosurg. Psychiatry 2020, 91, 271-277. [CrossRef] [PubMed]

7. Havrdova, E.; Galetta, S.; Hutchinson, M.; Stefoski, D.; Bates, D.; Polman, C.H.; O'Connor, P.W.; Giovannoni, G.; Phillips, J.T.; Lublin, F.D.; et al. Effect of natalizumab on clinical and radiological disease activity in multiple sclerosis: A retrospective analysis of the Natalizumab Safety and Efficacy in Relapsing-Remitting Multiple Sclerosis (AFFIRM) study. Lancet Neurol. 2009, 8, 254-260. [CrossRef]

8. Kappos, L.; Radue, E.-W.; O'Connor, P.; Polman, C.; Hohlfeld, R.; Calabresi, P.; Selmaj, K.; Agoropoulou, C.; Leyk, M.; ZhangAuberson, L.; et al. A Placebo-Controlled Trial of Oral Fingolimod in Relapsing Multiple Sclerosis. N. Engl. J. Med. 2010, 362, 387-401. [CrossRef] [PubMed]

9. Calabresi, P.A.; Radue, E.-W.; Goodin, D.; Jeffery, D.; Rammohan, K.W.; Reder, A.T.; Vollmer, T.; Agius, M.A.; Kappos, L.; Stites, T.; et al. Safety and efficacy of fingolimod in patients with relapsing-remitting multiple sclerosis (FREEDOMS II): A double-blind, randomised, placebo-controlled, phase 3 trial. Lancet Neurol. 2014, 13, 545-556. [CrossRef]

10. Río, J.; Rovira, A.; Tintoré, M.; Sastre-Garriga, J.; Castilló, J.; Auger, C.; Nos, C.; Comabella, M.; Tur, C.; Vidal, Á.; et al. Evaluating the response to glatiramer acetate in relapsing-remitting multiple sclerosis (RRMS) patients. Mult. Scler. J. 2014, 20, 1602-1608. [CrossRef] [PubMed]

11. Sormani, M.P.; Rio, J.; Tintorè, M.; Signori, A.; Li, D.; Cornelisse, P.; Stubinski, B.; Stromillo, M.L.; Montalban, X.; De Stefano, N. Scoring treatment response in patients with relapsing multiple sclerosis. Mult. Scler. J. 2013, 19, 605-612. [CrossRef] [PubMed]

12. Raffel, J.; Gafson, A.R.; Dahdaleh, S.; Malik, O.; Jones, B.; Nicholas, R. Inflammatory Activity on Natalizumab Predicts Short-Term but Not Long-Term Disability in Multiple Sclerosis. PLoS ONE 2017, 12, e0169546. [CrossRef] [PubMed]

13. Polman, C.H.; Reingold, S.C.; Banwell, B.; Clanet, M.; Cohen, J.A.; Filippi, M.; Fujihara, K.; Havrdova, E.; Hutchinson, M.; Kappos, L.; et al. Diagnostic criteria for multiple sclerosis: 2010 Revisions to the McDonald criteria. Ann. Neurol. $2011,69,292-302$. [CrossRef] [PubMed]

14. Meca-Lallana, J.; Ayuso, T.; Martínez-Yelamos, S.; Durán, C.; Martín, Y.C.; Navarro, N.H.; Sempere, A.P.; Álvarez-Cermeño, J.C.; Pascual, J.M.; Meca-Lallana, V.; et al. Effectiveness of Fingolimod versus Natalizumab as Second-Line Therapy for RelapsingRemitting Multiple Sclerosis in Spain: Second-Line GATE Study. Eur. Neurol. 2020, 83, 25-33. [CrossRef] [PubMed]

15. Curti, E.; Tsantes, E.; Baldi, E.; Caniatti, L.M.; Ferraro, D.; Sola, P.; Granella, F. The real-world effectiveness of natalizumab and fingolimod in relapsing-remitting multiple sclerosis. An Italian multicentre study. Mult. Scler. Relat. Disord. 2019, 33, 146-152. [CrossRef] [PubMed]

16. Baroncini, D.; Ghezzi, A.; Annovazzi, P.O.; Colombo, B.; Martinelli, V.; Minonzio, G.; Moiola, L.; Rodegher, M.; Zaffaroni, M.; Comi, G. Natalizumab versus fingolimod in patients with relapsing-remitting multiple sclerosis non-responding to first-line injectable therapies. Mult. Scler. J. 2016, 22, 1315-1326. [CrossRef] [PubMed] 
17. Preziosa, P.; Rocca, M.A.; Riccitelli, G.C.; Moiola, L.; Storelli, L.; Rodegher, M.; Comi, G.; Signori, A.; Falini, A.; Filippi, M. Effects of Natalizumab and Fingolimod on Clinical, Cognitive, and Magnetic Resonance Imaging Measures in Multiple Sclerosis. Neurotherapeutics 2020, 17, 208-217. [CrossRef] [PubMed]

18. Kapica-Topczewska, K.; Tarasiuk, J.; Collin, F.; Brola, W.; Chorąży, M.; Czarnowska, A.; Kwaśniewski, M.; Bartosik-Psujek, H.; Adamczyk-Sowa, M.; Kochanowicz, J.; et al. The effectiveness of interferon beta versus glatiramer acetate and natalizumab versus fingolimod in a Polish real-world population. PLoS ONE 2019, 14, e0223863. [CrossRef] [PubMed] 